\title{
Beneficios de la terapia psicológica y farmacológica en adultos con trastorno por atracón
}

Benefits of psychological and pharmacological therapy in adults with binge eating disorder

Brownley KA y col. Ann Intern Med. 2016;165(6):409-20.

\section{Objetivos}

Resumir la evidencia disponible sobre beneficios y daños de las terapias farmacológicas y psicológicas en adultos con trastorno por atracón.

\section{Fuente de datos}

Se realizaron búsquedas de estudios publicados en idioma inglés en MEDLINE (hasta mayo de 2016), EMBASE, Cochrane Library, Academic OneFile, CINAHL y ClinicalTrials.gov (hasta noviembre de 2015).

\section{Selección de Estudios}

Se incluyeron ensayos clínicos controlados y aleatorizados (ECCA) que incluyeran al menos 10 pacientes adultos con diagnóstico de trastorno por atracón basado en la cuarta o quinta edición del Manual Diagnóstico y Estadístico de los Trastornos Mentales (en inglés, DSM-IV o DSM-5). Las intervenciones evaluadas incluyeron tratamientos farmacológicos, psicológicos, conductuales y de medicinas alternativas o complementarias. Dos revisores independientes evaluaron los resúmenes y luego los textos completos de los artículos seleccionados, los desacu- erdos fueron resueltos por consenso o consulta con un tercer revisor.

\section{Extracción de Datos}

Los datos fueron extraídos por un revisor y luego cotejado por un segundo autor. El riesgo de sesgo de los ensayos incluidos fueron evaluados por dos revisores en forma independiente.

\section{Resultados Principales}

Se incluyeron 9 ensayos aleatorizados que evaluaron tratamiento psicológico vs control sin intervención psicológica y 25 ensayos aleatorizados controlados que evaluaron tratamiento farmacológico vs placebo $(n=19)$ o una combinación de tratamiento psicológico y farmacológico vs control o placebo $(n=6)$. Todos los ensayos tuvieron bajo o mediano riesgo de sesgo.

En la tabla 1 se resumen los resultados de los meta-análisis realizados para el desenlace principal: abstinencia de atracones. Los autores describen en forma narrativa que otros tratamientos que mostraron reducción de atracones fueron el topiramato, la terapia cognitivo conductual (TCC) parcialmente guiada por un terapeuta y la TCC auto-guiada por el paciente.

Tabla 1. Efectos de la terapia cognitivo conductual, la lisdexanfetamina y los antidepresivos de segunda generación vs. placebo sobre la abstinencia de atracones en pacientes con diagnóstico de trastorno por atracón.

\begin{tabular}{l|c|c|c}
\multicolumn{1}{c|}{ Intervenciones evaluadas } & RR (IC 95\%) & $\begin{array}{c}\text { Eventos grupo } \\
\text { tratamiento/n pacientes }\end{array}$ & $\begin{array}{c}\text { Eventos grupo } \\
\text { placebo/n pacientes }\end{array}$ \\
\hline TCC-GT & $4,95(3,06 \mathrm{a} 8,00)$ & $90 / 153$ & $16 / 142$ \\
\hline Lisdexanfetamina & $2,61(2,04 \mathrm{a} 3,33)$ & $208 / 517$ & $67 / 450$ \\
\hline Antidepresivos segunda generación & $1,67(1,24 \mathrm{a} 2,26)$ & $83 / 208$ & $49 / 208$ \\
\hline
\end{tabular}

RR: riesgo relativo; IC 95\%: intervalo de confianza del 95\%; TCC-GT: terapia cognitivo-conductual guiada por un terapeuta.

La lisdexanfetamina (en dosis de 50 a $70 \mathrm{mg} /$ día) y los antidepresivos de segunda generación (por ejemplo, citalopram, fluoxetina y sertralina) redujeron las obsesiones y compulsiones relacionadas con la alimentación (diferencia de medias o MD: -6,50 [IC $95 \%,-8,82$ a $-4,18]$ y MD: $-3,84$ [-6,55 a $-1,13]$, respectivamente), mientras que estos últimos agentes redujeron además los síntomas depresivos medidos a través de la escala de Hamilton (MD, $-1,97[-3,67$ a $-0,28])$.

Síntomas adversos como dolor de cabeza, malestar gastrointestinal, trastornos del sueño y excitación del sistema nervioso simpático ocurrieron con mayor frecuencia en los pacientes que recibieron lisdexanfetamina comparados con quienes recibieron placebo (rango de riesgo relativo entre 1,63 y 4,28 ).

\section{Conclusiones}

La TCC, los antidepresivos de segunda generación, la lisdexanfetamina y los anticonvulsivantes como el topiramato reducen los episodios de atracones y sus patologías psicológicas asociadas.

Fuente de Financiamiento: agencia de investigación y calidad de la asistencia sanitaria (Agency for Healthcare Research and Quality).

\section{Comentario}

El trastorno por atracón, el más frecuente de los desórdenes alimentarios (aproximadamente 3\% de prevalencia), y según criterios de la Asociación Norteamericana de Psiquiatría (en inglés, American Psychiatric Association o APA) publicados en el DSM-5 ${ }^{1}$, se caracteriza por: episodios recurrentes (al menos 1 por semana durante 3 meses), psicológicamente angustiantes, de breve duración (de 2 horas aproximadamente), durante los cuales los pacientes sienten una falta de control y consumen grandes cantidades de alimentos (cantidades mayores que la mayoría de las personas consumirían en el mismo período). Otros criterios diagnósticos son: ingestas de grandes cantidades de alimento, más rápido que lo normal, inclusive con sensación de saciedad, a escondidas, y acompañados de sensación de culpabilidad o depresión. Estas conductas no están asociadas a comportamientos compensatorios inapropiados. Este problema es más frecuente en mujeres $(3,5 \%)$ que en hombres $(2,0 \%)$ y en personas obesas $(5 \% \text { a } 30 \%)^{2-3}$. Por lo general surge en la adultez temprana y se asocia con trastornos psiquiátricos, dolor crónico, obesidad y diabetes. Las guías actuales de la APA $^{1}$ y del Instituto Nacional para la Salud y Cuidado de la Excelencia ${ }^{4}$ (NICE, por sus iniciales en inglés) apoyan el uso de la TCC y los inhibidores selectivos de la recaptación de serotonina. La APA recomienda un enfoque de equipo (incluyendo psiquiatras, psicólogos, dietistas y trabajadores sociales) con la TCC como piedra angular del tratamiento, y el uso de fármacos como tratamiento adyuvante.

Por el contrario, NICE recomienda el enfoque de autoayuda basado en la TCC, pero también respalda el uso de medicación como monoterapia para algunos pacientes. Una de las limitaciones del estudio fue que la mayoría de los participantes eran mujeres blancas con sobrepeso u obesas de entre 20 y 40 años. Muchos tratamientos fueron examinados solo en estudios individuales. Los resultados fueron medidos inconsistentemente en los ensayos y raramente evaluados más allá del final del tratamiento. 


\section{Conclusiones de las comentadoras}

El tratamiento de este trastorno tiene como objetivos reducir la frecuencia de atracones, mejorar la salud metabólica y el peso corporal, y regular el estado de ánimo en pacientes con depresión o ansiedad coexistente. Los enfoques de tratamiento incluyen tratamiento psicológico cognitivo conductual, farmacológico o la combinación de ambos. En nuestra experiencia el abordaje terapéutico requiere de la expertise de los profesionales actuantes para adecuar el tratamiento a cada caso utilizando los diferentes abordajes según el caso.

Susana Ruth Gutt [ Sección Nutrición - Servicio de Clínica Médica, Hospital Italiano de Buenos Aires. susana.gutt@ hospitalitalaino.org.ar ]

Analía Verónica Venczel [ Sección Nutrición - Servicio de Clínica Médica, Hospital Italiano de Buenos Aires. analia.venczel@hospitalitaliano.org.ar ]

Gutt, S y Venczel, A. Beneficios de la terapia psicológica y farmacológica en adultos con trastorno por atracón. Evid Actual Pract Ambul. 2018;21(3):7677. Comentado de: Brownley KA y col, Binge-eating disorder in adults: a systematic review and meta-analysis. Annals of Internal Medicine 2016;165 (6) 409-20 PMID: 27367316.

\section{Referencia}

1. American Psychiatric Association. Diagnostic and Statistical Manual of Mental Disorders. 5th ed. 2013.

2. Bruce $B$ y col. Binge eating among the overweight population: a serious and prevalent problem. J Am Diet Assoc. 1996; 96:58-61.

3. Spitzer R y col. Binge eating disorder: its further validation in a multisite study. Int Eat Disord 1993;13: 137-153.

4. National Institute for Health and Care Excellence (NICE). Eating disorders in over 8s: management. Guidance. 2014. Disponible en: https://www.nice. org.uk/guidance/cg9

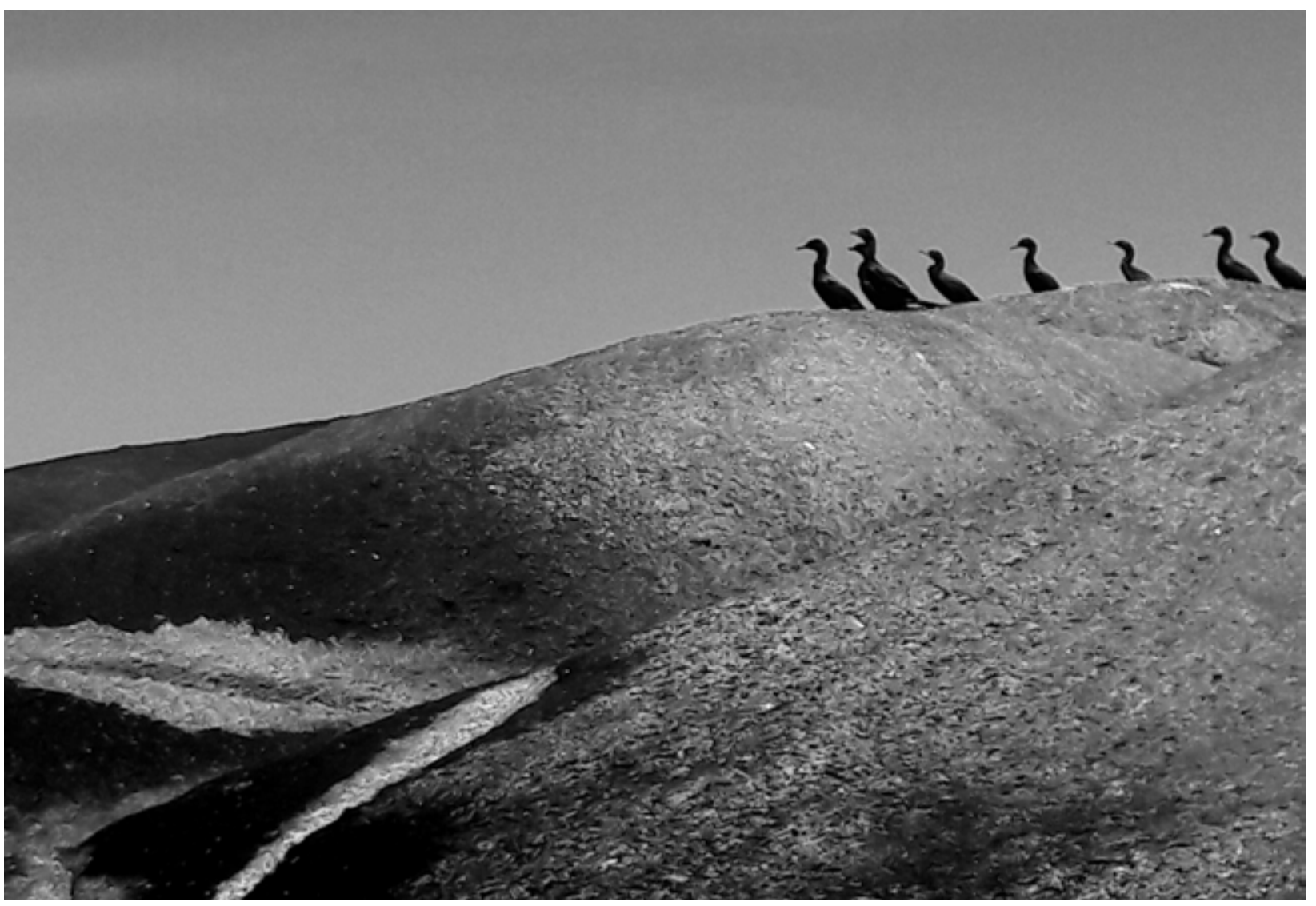

Cortesía de Silvia Spina 